\title{
Bis(phenyl)dirhodium(III) Caprolactamate: A Dinuclear Paddlewheel Complex With No Metal-Metal Bond
}

\author{
Jason M. Nichols, ${ }^{\ddagger}$ Joffrey Wolf, ${ }^{\S}$ Peter Y. Zavalij, ${ }^{\ddagger}$ Bindhu Varughese, ${ }^{\ddagger}$ and Michael P. Doyle ${ }^{\ddagger}$ \\ ${ }^{\ddagger}$ Department of Chemistry and Biochemistry, University of Maryland, College Park, MD 20742 \\ ${ }^{\S}$ Laboratoire de Chimie de Coordination, 205 route de Narbonne, 31077 Toulouse Cedex 4, France.
}

\section{Experimental Section}

General. All reagents were commercially obtained and used without further purification. ${ }^{1} \mathrm{H}$ NMR $(400 \mathrm{MHz})$ and ${ }^{13} \mathrm{C}$ NMR (100 MHz) spectra were obtained on a Bruker DRX-400 NMR as solutions in $\mathrm{CDCl}_{3}$. Chemical shifts are reported in parts per million (ppm, $\varepsilon$ ) downfield from $\mathrm{Me}_{4} \mathrm{Si}$ (TMS). Electrospray and high resolution mass spectra were performed on a JEOL AccuTOF-CS (ESI) or a JEOL SX102 (FAB) instrument. UV/Visible spectra were obtained on a Varian Cary 50 spectrophotometer using a xenon flash lamp. IR spectra were recorded on a JASCO FT/IR 4100 spectrometer. All preparatory silica gel columns were run with Silicycle Ultrapure silica (230-400 mesh).

XRD Crystal Structure Determination. Data were collected using SMART. ${ }^{2}$ Cell determination, refinement and frames integration were done with the SAINT software package using a narrow-frame integration algorithm. $^{2}$ The X-ray intensity data were measured at $223(2){ }^{\circ} \mathrm{K}$ on a three-circle diffractometer system equipped with Bruker Smart 1000 CCD area detector using a graphite monochromator and a MoK $\alpha$ fine-focus sealed tube $(\lambda=0.71073 \AA$ ) operated at $50 \mathrm{kV}$ and $40 \mathrm{~mA}$. The detector was placed at a distance of $4.958 \mathrm{~cm}$ from the crystal. Data were corrected for absorption effects with the Semi-empirical from equivalents method using $\mathrm{XPREP}^{3}$ for $\mathbf{1}$ or SADABS for $\mathbf{2}$ and $\mathbf{3}^{4}$ Structures were solved and refined using the SHELXS-97 and SHELXL-97 software. ${ }^{4,5}$

XPS Binding Energy Determination. A Kratos Axis 165 X-ray photoelectron spectrometer was used to determine the $\mathrm{Rh} 3 d$ binding energies in compounds $\mathbf{1}, \mathbf{2}$ and $\mathbf{3}$ at a pressure of $8 \times 10^{-10}$ Torr with nonmonochromatic $\mathrm{Mg} \mathrm{K}$ radiation. The powder samples were dusted on to double-sided conductive carbon tape mounted on a sample stub. High-resolution measurements for various regions (Rh, N, O, F, S and C) were done in hybrid mode using both electrostatic and magnetic lenses, with a step size of $0.1 \mathrm{eV}$ at a pass energy of 40 $\mathrm{eV}$. The charge neutralizer was on during the measurements to compensate surface charging and binding energies were calibrated with respect to $\mathrm{C} 1 \mathrm{~s}$ at $284.6 \mathrm{eV}$.

XPS Data Analysis. Data processing was performed using Vision processing software. After subtraction of a linear background, all spectra are fitted using 60\% Gaussian/40\% Lorentzian peaks, taking the minimum number of peaks consistent with the best fit. The important parameters used for this fitting are peak position, full width at half maximum, and spin orbit coupling. 
tetrakis- $\mu$-( $\varepsilon$-caprolactamato)-bis(acetonitrile)dirhodium(II) [2].

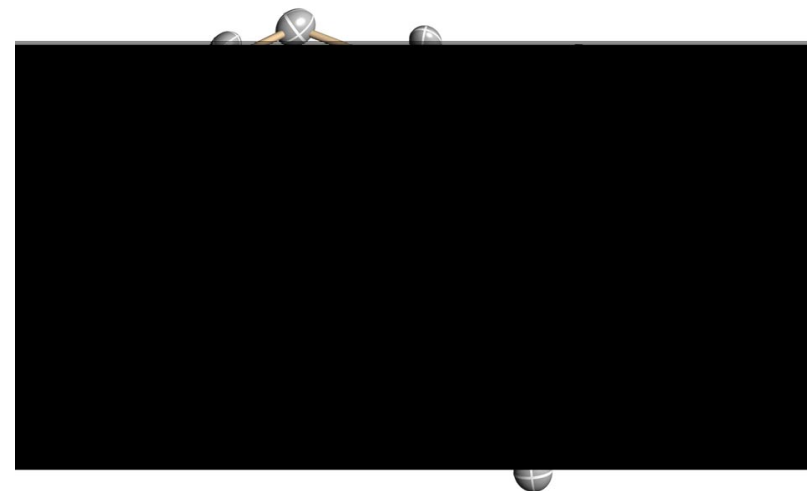

ORTEP drawing of $2, \mathrm{C}_{32} \mathrm{H}_{52} \mathrm{~N}_{8} \mathrm{O}_{4} \mathrm{Rh}_{2}, \mathrm{R} 1=4.29 \%$. Selected bond lengths $(\AA)$ and angles (deg): $\mathrm{Rh} 1-\mathrm{Rh} 2$ (2.422); Rh1-N1 (2.336) ; Rh2-O11 (2.050); Rh1-N11 (2.038); Rh2-Rh1-N1 (174.4); Rh2-Rh1-N11 (86.7); Rh1-Rh2-O11 (89.6); Rh2-Rh1-N21 (86.9); Rh1-Rh2-O21 (89.3). CCDC \#615575. Ellipsoids are shown at a $30 \%$ probability level. Solvent molecules and hydrogens are omitted for clarity.

The preparation of dirhodium(II) caprolactamate $\left[\mathrm{Rh}_{2}(\mathrm{cap})_{4} \cdot 2 \mathrm{CH}_{3} \mathrm{CN}\right]$ has been previously described. ${ }^{1}$ Synthetic and X-ray quality material was provided by recrystallization. Recrystallization procedure: An oven dried 250 $\mathrm{mL}$, single-necked (24/40 joint) round-bottomed flask, equipped with a Teflon-coated stir-bar was charged with $3.52 \mathrm{~g}$ of $\mathrm{Rh}_{2}(\mathrm{cap})_{4}, 100 \mathrm{~mL}$ of acetonitrile, and $10 \mathrm{~mL}$ of methanol. The flask was placed in an oil bath maintained at $100^{\circ} \mathrm{C}$. When the mixture reached boiling, methanol was added sparingly via Pasteur pipette until a deep purple homogenous solution was obtained. Care was taken to avoid excess methanol. The solution was removed from the oil bath and filtered hot over a funnel containing a small cotton plug. The solution was allowed to cool to room temperature and was placed into the freezer overnight. Shiny purple crystals were isolated by removal of the supernatant liquid. The crystals were washed with acetonitrile $(3 \times 5 \mathrm{~mL})$ and dried for 10 minutes at $0.1 \mathrm{~mm} \mathrm{Hg}$ to provide $\mathrm{Rh}_{2}(\mathrm{cap})_{4} \cdot 2 \mathrm{CH}_{3} \mathrm{CN}$ as shiny deep purple, $\mathrm{X}$-Ray quality crystals.

Anal. calc'd. for $\mathrm{Rh}_{2} \mathrm{C}_{28} \mathrm{H}_{46} \mathrm{~N}_{6} \mathrm{O}_{4}$ (736.52): $\mathrm{C}(45.66), \mathrm{H}(6.29)$, $\mathrm{N}(11.41)$ / Found $\mathrm{C}(45.65), \mathrm{H}(6.44), \mathrm{N}(11.90) ;{ }^{1} \mathrm{H}$ NMR (400 MHz, CDCl $)$ \& 3.24-3.37 (m, 8H, N-CH $)$, 2.31-2.41 (m, 8H, C-CH $)_{2}$, 2.07 (br, 9H), 1.39-1.67 (m, $\left.24 \mathrm{H}, \mathrm{N}-\mathrm{CH}_{2}-\mathrm{CH}_{2}-\mathrm{CH}_{2}-\mathrm{CH}_{2}\right)$ ppm; ${ }^{13} \mathrm{C}$ NMR $\left(100.6 \mathrm{MHz}, \mathrm{CDCl}_{3}\right) \& 185.7,115.6,53.6,36.7,30.6,28.9,24.1$, $2.3 \mathrm{ppm}$; UV/Visible (MeCN/MeOH 1:1) $362 \mathrm{~nm}\left(\varepsilon=174 \mathrm{M}^{-1} \mathrm{~cm}^{-1}\right), 516 \mathrm{~nm}\left(\varepsilon=188 \mathrm{M}^{-1} \mathrm{~cm}^{-1}\right)$; HRMS (FAB) calc'd for $\mathrm{Rh}_{2} \mathrm{C}_{24} \mathrm{H}_{40} \mathrm{~N}_{4} \mathrm{O}_{4} 654.1160$, found $654.1180(\mathrm{M}+)$. 
tetrakis- $\mu-(\varepsilon$-caprolactamato) bis(aquo)dirhodium(II/III) triflate, [3].

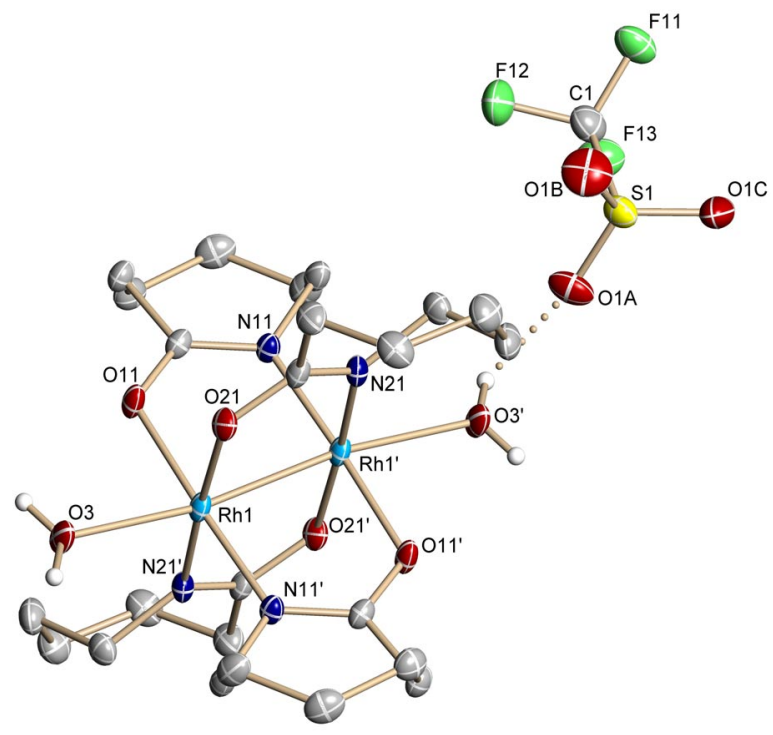

ORTEP drawing of $3, \mathrm{C}_{25} \mathrm{H}_{44} \mathrm{~F}_{3} \mathrm{~N}_{4} \mathrm{O}_{9} \mathrm{Rh}_{2} \mathrm{~S}, \mathrm{R} 1=3.72 \%$. Selected bond lengths ( $\mathrm{\AA}$ ) and angles (deg): Rh1-Rh1' (2.384); Rh1'-O3' (2.288), Rh1-O11 (2.023); Rh1'-N11 (1.996); Rh1-Rh1'-O3' (171.3); Rh1-Rh'-N1 (88.4); Rh1'-Rh1-O11 (88.6); Rh1-Rh1'-N21 (88.6); Rh1'-Rh1-O21 (88.5). CCDC \#615576. Ellipsoids are shown at a $30 \%$ probability level. Hydrogens are omitted for clarity.

A $125 \mathrm{~mL}$ Erlenmeyer flask was charged with freshly crushed $2(25 \mathrm{mg}, 0.034 \mathrm{mmols}), 20 \mathrm{~mL}$ of ethyl acetate was added, and the resulting mixture was sonicated for 10 minutes until a fine powder suspension was observed. Copper(II) triflate (13 mg, $0.036 \mathrm{mmols}$ ) was added, and the ethyl acetate reaction mixture was heated to boiling until a translucent purple solution was obtained. The solution was allowed to cool to room temperature, and solvent was removed in vacuo to yield a purple glassy solid. Recrystallization from $\mathrm{CH}_{2} \mathrm{Cl}_{2}$ with slow diffusion of diethyl ether yielded X-ray quality crystals. Yield: $15 \mathrm{mg}(53 \%)$.

NMR silent; UV/Visible $\left(\mathrm{CH}_{2} \mathrm{Cl}_{2}\right) 505 \mathrm{~nm}\left(\varepsilon=4501 \mathrm{M}^{-1} \mathrm{~cm}^{-1}\right), 970 \mathrm{~nm}\left(\varepsilon=1087 \mathrm{M}^{-1} \mathrm{~cm}^{-1}\right)$. 


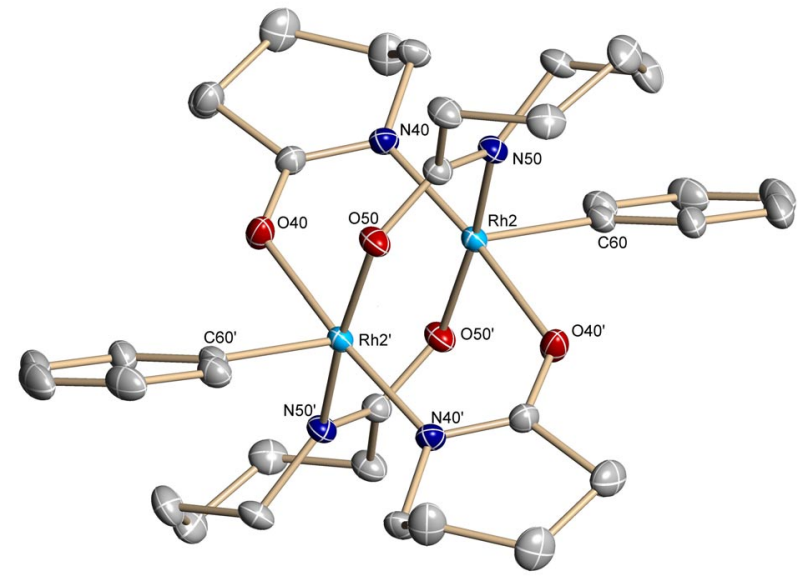

ORTEP drawing of one of two crystallographically independent molecules of $1, \mathrm{C}_{36} \mathrm{H}_{50} \mathrm{~N}_{4} \mathrm{O}_{4} \mathrm{Rh}_{2}, \mathrm{R}_{1}=3.00 \%$. Selected bond lengths (Å) and angles (deg): Rh2-Rh2' (2.519); Rh2-C3B (2.000), Rh2'-O41 (2.085); Rh2'-O51 (2.083); Rh2-N41 (2.011); Rh2-N51 (2.014); Rh2-Rh2'-C3B' (155.4); Rh2'-Rh2-N41 (96.4); Rh2'-Rh2-N51 (94.0); Rh2-Rh2'-O41 (77.4); Rh2-Rh2'-O51 (79.8). CCDC \#615577. Ellipsoids are shown at a 30\% probability level. Solvent and hydrogens are omitted for clarity.

In a $25 \mathrm{~mL}$ round bottom flask, $\mathrm{NaBPh}_{4}(163 \mathrm{mg}, 476 \mu \mathrm{mol})$ and $\mathrm{Cu}(\mathrm{I}) \mathrm{OTf} \cdot \mathrm{C}_{6} \mathrm{H}_{6}(3 \mathrm{mg}, 11 \mu \mathrm{mol})$ were added to a $\mathrm{CH}_{2} \mathrm{Cl}_{2} / \mathrm{MeOH}$ solution (10 mL, 9:1) of 2 (70 mg, $\left.95 \mu \mathrm{mol}\right)$. During the first minutes of reaction, the solution color turned from red-purple to green after which the reaction was left to stir at room temperature under air. At 15 hours, the reaction was concentrated to dryness, loaded onto a silica gel column, and purified via gradient elution using pentane $/ \mathrm{CH}_{2} \mathrm{Cl}_{2}(5: 1,100 \mathrm{~mL}) \rightarrow \mathrm{CH}_{2} \mathrm{Cl}_{2}(50 \mathrm{~mL}) \rightarrow \mathrm{CH}_{2} \mathrm{Cl}_{2} /$ acetone $(95: 5,50 \mathrm{~mL})$. The desired fractions were concentrated to a dark green solid that was triturated with diethyl ether $(2 \times 5 \mathrm{~mL})$, filtered, and dried in vacuo. Yield: $59 \mathrm{mg}(77 \%)$. Recrystallization from $\mathrm{CH}_{2} \mathrm{Cl}_{2}$ provided X-ray quality crystals.

Anal. calc'd. for $\mathrm{Rh}_{2} \mathrm{C}_{36} \mathrm{H}_{51} \mathrm{~N}_{4} \mathrm{O}_{4}$ (809.20): C(53.47), H(6.23), N(6.93) / Found: $\mathrm{C}(53.04), \mathrm{H}(6.15), \mathrm{N}(6.63)$; ${ }^{1} \mathrm{H}$ NMR $\left(500 \mathrm{MHz}, 20^{\circ} \mathrm{C}, \mathrm{CD}_{2} \mathrm{Cl}_{2}\right): \varepsilon$ 7.49-7.55 (m, 4H, o-Ph), 7.11-7.18 (m, 6H, m,p-Ph), 3.00-3.10 (m, 8H, N$\left.\mathrm{CH}_{2}\right), 2.48-2.53$ (m, 4H, C- $\left.\mathrm{CH}_{2}\right), 2.40-2.44$ (m, 4H, C-CH$), 1.78-2.85$ (m, 4H, N-CH $\left.-\mathrm{CH}_{2}\right), 1.45-1.70(\mathrm{~m}, 20 \mathrm{H}$, $\left.\mathrm{N}-\mathrm{CH}_{2}-\left(\mathrm{CH}_{2}\right)_{3}\right) \mathrm{ppm} ;{ }^{13} \mathrm{C} \mathrm{NMR}\left(125.8 \mathrm{MHz}, 20^{\circ} \mathrm{C}, \mathrm{CD}_{2} \mathrm{Cl}_{2}\right) \varepsilon 183.72,147.80\left(\mathrm{dd},{ }^{1} J_{\mathrm{C}-\mathrm{Rh}}=36.4 \mathrm{~Hz} ;{ }^{2} J_{\mathrm{C}-\mathrm{Rh}}=\right.$ 1.6Hz, $\left.\mathrm{C}_{\text {ipso }} \mathrm{Ph}\right), 137.15,126.18,123.99,51.44,38.63,30.71,29.81,24.37 \mathrm{ppm}$; UV/Visible $\left(\mathrm{CH}_{2} \mathrm{Cl}_{2}\right): \lambda_{\max }$ $\left(\varepsilon \mathrm{M}^{-1} \mathrm{~cm}^{-1}\right)=430 \mathrm{~nm} \quad(4540) ; \quad \operatorname{IR}($ neat$): \quad v=1550(\mathrm{~s}), \quad 1583(\mathrm{~s}) \mathrm{cm}^{-1} ;$ HRMS (ESI) calc'd for $\mathrm{Rh}_{2} \mathrm{C}_{36} \mathrm{H}_{51} \mathrm{~N}_{4} \mathrm{O}_{4}$ 809.2020, found 809.2018 (M+H). 
Table 1S: Comparison of selected angles $\left({ }^{\circ}\right)$, torsions $\left({ }^{\circ}\right)$ and bond distances $(\AA)$ between compounds $\mathbf{1}, \mathbf{2}, \mathbf{3}$.

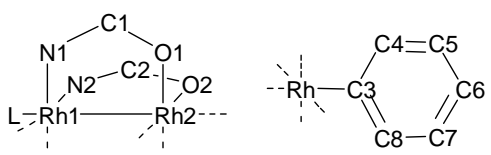

\begin{tabular}{|l|l|l|l|}
\hline & $\mathbf{2}$ & $\mathbf{3}$ & $\mathbf{1}$ \\
\hline (a) Bond Distances & & & \\
$\mathrm{Rh}(1)-\mathrm{Rh}(2)$ & $2.4221(4)$ & $2.3840(6)$ & $2.5188(3)$ \\
$\mathrm{Rh}(2)-\mathrm{O}(1)$ & $2.050(2)$ & $2.023(2)$ & $2.0825(16)$ \\
$\mathrm{Rh}(2)-\mathrm{O}(2)$ & $2.053(2)$ & $2.030(2)$ & $2.0777(16)$ \\
$\mathrm{Rh}(1)-\mathrm{N}(1)$ & $2.038(3)$ & $1.988(3)$ & $2.012(2)$ \\
$\mathrm{Rh}(1)-\mathrm{N}(2)$ & $2.045(3)$ & $1.996(3)$ & $2.0078(19)$ \\
$\mathrm{C}(1)-\mathrm{O}(1)$ & $1.292(4)$ & $1.291(4)$ & $1.287(3)$ \\
$\mathrm{C}(2)-\mathrm{O}(2)$ & $1.290(4)$ & $1.296(4)$ & $1.290(3)$ \\
$\mathrm{C}(1)-\mathrm{N}(1)$ & $1.309(4)$ & $1.312(4)$ & $1.311(3)$ \\
$\mathrm{C}(2)-\mathrm{N}(2)$ & $1.313(4)$ & $1.311(4)$ & $1.304(3)$ \\
$\mathrm{Rh}-\mathrm{L}_{\text {axial }}$ & $2.336(3)$ & $2.288(2)$ & $2.010(2)$ \\
& & & \\
\hline (b) Bond Angles & & & \\
$\mathrm{Rh}(1)-\mathrm{Rh}(2)-\mathrm{O}(1)$ & $89.62(6)$ & $88.58(7)$ & $79.29(4)$ \\
$\mathrm{Rh}(1)-\mathrm{Rh}(2)-\mathrm{O}(2)$ & $89.37(6)$ & $88.50(7)$ & $78.39(5)$ \\
$\mathrm{Rh}(2)-\mathrm{Rh}(1)-\mathrm{N}(1)$ & $86.70(7)$ & $88.42(8)$ & $94.46(5)$ \\
$\mathrm{Rh}(2)-\mathrm{Rh}(1)-\mathrm{N}(2)$ & $86.81(7)$ & $88.60(8)$ & $95.32(5)$ \\
$\mathrm{Rh}(2)-\mathrm{O}(1)-\mathrm{C}(1)$ & $118.3(2)$ & $119.8(2)$ & $128.11(15)$ \\
$\mathrm{Rh}(2)-\mathrm{O}(2)-\mathrm{C}(2)$ & $118.96(19)$ & $119.5(2)$ & $128.97(15)$ \\
$\mathrm{Rh}(1)-\mathrm{N}(1)-\mathrm{C}(1)$ & $121.5(2)$ & $120.8(2)$ & $114.45(16)$ \\
$\mathrm{Rh}(1)-\mathrm{N}(2)-\mathrm{C}(2)$ & $121.7(2)$ & $121.2(2)$ & $113.51(15)$ \\
$\mathrm{O}(1)-\mathrm{C}(1)-\mathrm{N}(1)$ & $123.2(3)$ & $122.3(3)$ & $123.6(2)$ \\
$\mathrm{O}(2)-\mathrm{C}(2)-\mathrm{N}(2)$ & $123.2(3)$ & $122.2(3)$ & $123.8(2)$ \\
$\mathrm{Rh}(1)-\mathrm{Rh}(2)-\mathrm{L}_{\text {axial }}$ & $174.42(7)$ & $171.28(7)$ & $156.19(7)$ \\
& & & \\
\hline (c) Torsion angles & & & \\
$\mathrm{O}(1)-\mathrm{Rh}-\mathrm{Rh}-\mathrm{N}(1)$ & 0.61 & 0.65 & 1.42 \\
$\mathrm{O}(2)-\mathrm{Rh}-\mathrm{Rh}-\mathrm{N}(2)$ & 1.05 & 0.73 & 1.78 \\
\hline
\end{tabular}

Table 2S: Phenyl C-C bondlenghs ( $(\AA)$ for one of two crystallographically independent structures of $\mathbf{3}$.

\begin{tabular}{|l|l|l|l|l|l|}
\hline C3-C4 & C3-C8 & C4-C5 & C8-C7 & C5-C6 & C7-C6 \\
\hline $1.390(4)$ & $1.397(4)$ & $1.390(4)$ & $1.391(4)$ & $1.374(4)$ & $1.372(4)$ \\
\hline
\end{tabular}


Table 3S. XPS data for $\mathbf{2}$ with selected peak graphs.

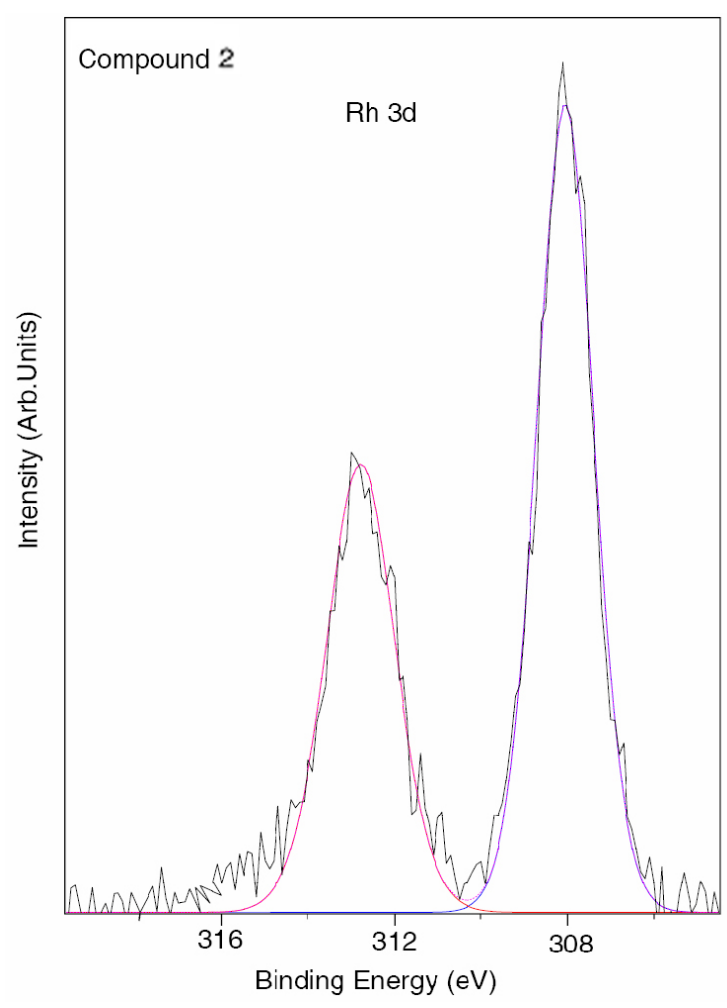

Table 4S. XPS data for 3 with selected peak graphs.

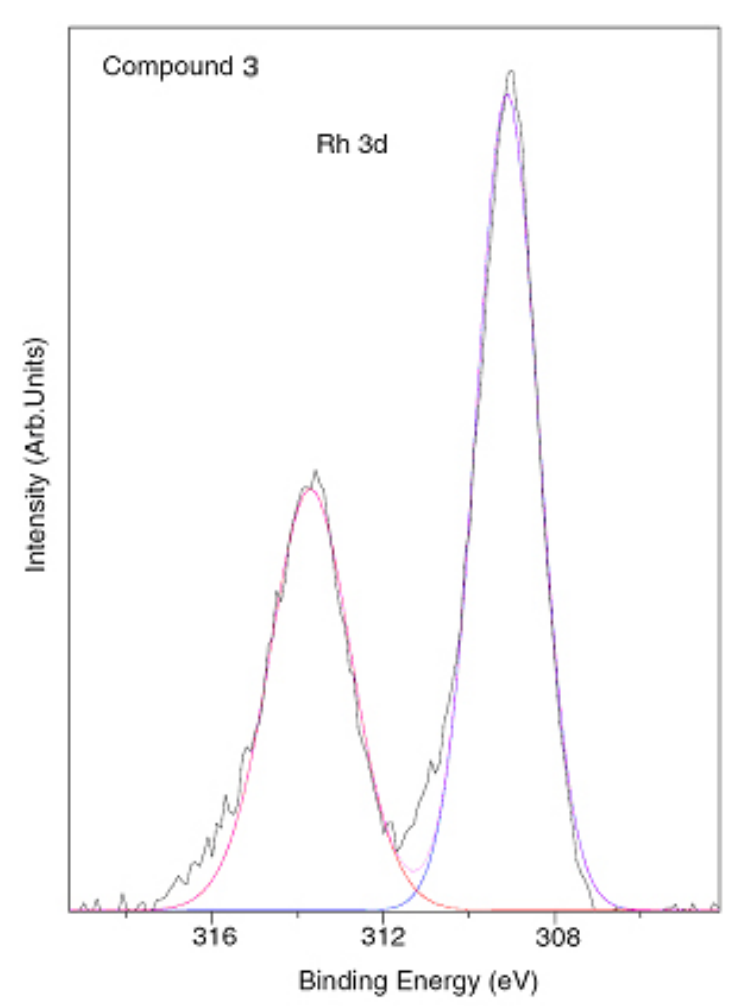

\begin{tabular}{|l|l|l|l|}
\hline Peak & $\begin{array}{l}\text { Position } \\
(\mathbf{e V})\end{array}$ & $\begin{array}{l}\text { FWHM } \\
(\mathbf{e V})\end{array}$ & $\begin{array}{l}\text { Atomic } \\
\text { Conc \% }\end{array}$ \\
\hline $\mathrm{Rh} 3 d 5 / 2$ & 308.080 & 1.532 & 2.05 \\
$\mathrm{Rh} 3 d 3 / 2$ & 312.812 & 1.838 & 1.36 \\
$\mathrm{~N} 1 s \mathrm{a}$ & 397.874 & 1.577 & 6.51 \\
$\mathrm{~N} 1 s \mathrm{~b}$ & 399.284 & 1.626 & 1.58 \\
$\mathrm{O} 1 s \mathrm{a}$ & 530.982 & 1.878 & 10.54 \\
$\mathrm{O} 1 s \mathrm{~b}$ & 532.832 & 2.144 & 4.88 \\
$\mathrm{C} 1 s \mathrm{a}$ & 284.600 & 1.611 & 55.91 \\
$\mathrm{C} 1 s \mathrm{~b}$ & 286.259 & 1.334 & 10.10 \\
$\mathrm{C} 1 s \mathrm{c}$ & 288.029 & 2.311 & 7.07 \\
\hline
\end{tabular}

\begin{tabular}{|l|l|l|l|}
\hline Peak & $\begin{array}{l}\text { Position } \\
(\mathbf{e V})\end{array}$ & $\begin{array}{l}\text { FWHM } \\
(\mathbf{e V})\end{array}$ & $\begin{array}{l}\text { Atomic } \\
\text { Conc \% }\end{array}$ \\
\hline $\mathrm{Rh} 3 d 5 / 2$ & 309.094 & 1.578 & 1.68 \\
$\mathrm{Rh} 3 d 3 / 2$ & 313.718 & 2.202 & 1.32 \\
$\mathrm{~N} 1 s \mathrm{a}$ & 398.282 & 1.600 & 5.79 \\
$\mathrm{~N} 1 s \mathrm{~b}$ & 400.105 & 1.950 & 0.89 \\
$\mathrm{O} 1 s \mathrm{a}$ & 531.120 & 1.692 & 14.15 \\
$\mathrm{O} 1 s \mathrm{~b}$ & 532.534 & 1.525 & 3.81 \\
$\mathrm{O} 1 s \mathrm{c}$ & 534.018 & 1.580 & 1.27 \\
$\mathrm{C} 1 s \mathrm{a}$ & 284.600 & 1.708 & 47.21 \\
$\mathrm{C} 1 s \mathrm{~b}$ & 286.471 & 1.981 & 13.15 \\
$\mathrm{C} 1 s \mathrm{c}$ & 291.874 & 1.233 & 1.68 \\
$\mathrm{C} 1 s \mathrm{~d}$ & 288.851 & 1.879 & 2.23 \\
$\mathrm{~F} 1 s \mathrm{a}$ & 687.470 & 1.719 & 3.98 \\
$\mathrm{~F} 1 s \mathrm{~b}$ & 688.801 & 1.648 & 1.14 \\
$\mathrm{~S} 2 p 3 / 2$ & 167.505 & 1.859 & 0.99 \\
$\mathrm{~S} 2 p 1 / 2$ & 168.685 & 1.859 & 0.69 \\
\hline
\end{tabular}


Table 5S. XPS data for 1 with selected peak graphs.

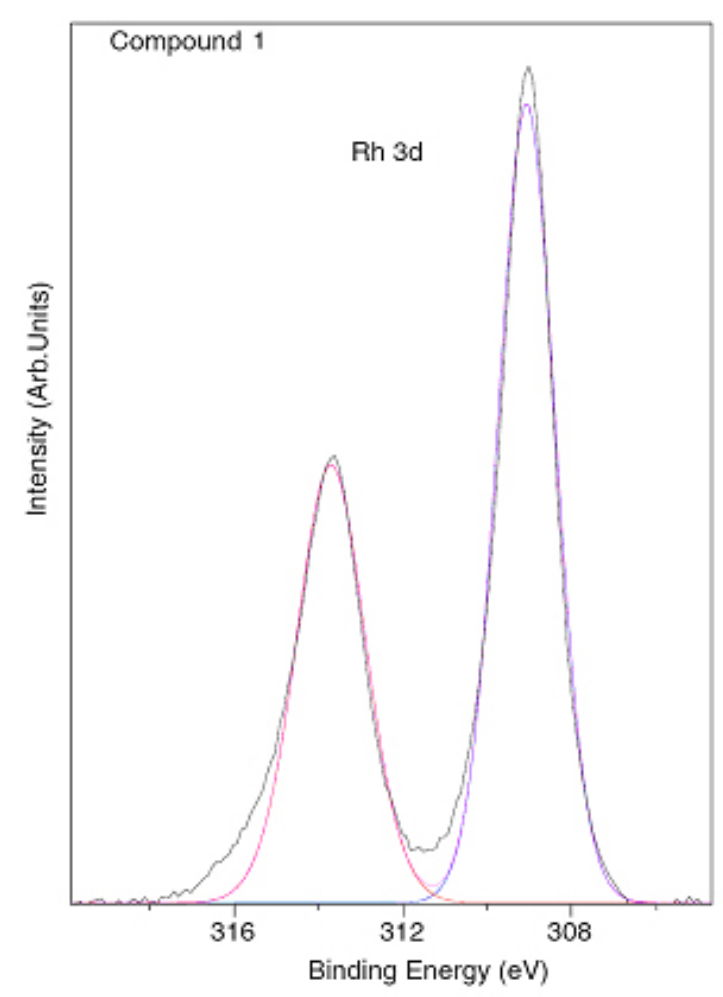

\begin{tabular}{|l|l|l|l|}
\hline Peak & $\begin{array}{l}\text { Position } \\
(\mathbf{e V})\end{array}$ & $\begin{array}{l}\text { FWHM } \\
(\mathbf{e V})\end{array}$ & $\begin{array}{l}\text { Atomic } \\
\text { Conc \% }\end{array}$ \\
\hline Rh 3d 5/2 & 309.105 & 1.571 & 1.10 \\
$\mathrm{Rh} \mathrm{3d} 3 / 2$ & 313.725 & 1.892 & 0.72 \\
$\mathrm{~N} 1 s \mathrm{a}$ & 398.290 & 1.405 & 3.94 \\
$\mathrm{~N} 1 s \mathrm{~b}$ & 399.783 & 1.436 & 0.49 \\
$\mathrm{O} 1 s \mathrm{a}$ & 531.210 & 1.911 & 6.77 \\
$\mathrm{O} 1 s \mathrm{~b}$ & 532.934 & 2.050 & 4.07 \\
$\mathrm{C} 1 s \mathrm{a}$ & 284.610 & 1.613 & 69.96 \\
$\mathrm{C} 1 s \mathrm{~b}$ & 286.404 & 1.498 & 11.09 \\
$\mathrm{C} 1 s \mathrm{c}$ & 288.289 & 1.697 & 1.85 \\
\hline
\end{tabular}

\section{References.}

[1] Doyle, M. P.; Westrum, L. J.; Wolthuis, W. N. E.; See, M. M.; Boone, W. P.; Bagheri, V.; Pearson, M. M. J. Am. Chem. Soc. 1993, 115, 958.

[2] Bruker (1999). SMART and SAINT. Bruker AXS Inc., Madison, Wisconsin, USA.

[3] Sheldrick, G. M. (1997). SHELXL-97. University of Göttingen, Germany.

[4] Sheldrick, G. M. (1996). SADABS. University of Göttingen, Germany.

[5] Sheldrick, G. M. (1990). Acta Cryst. A46, 467-473. 